\title{
Modelagem Matemática da Curva de Descarga da Bateria de um Quadrirotor Rolling Spider Utilizando a Teoria de Identificação de Sistemas
}

\author{
Adriel de O. Freitas, Vandilberto P. Pinto, \\ Camila Tabosa de S. Lima, Ligia R. Machado, \\ Francisco Alexandro S. Freitas
Universidade Federal do Ceará, Campus de Sobral, Rua Coronel Estanislau Frota s/n, Sobral-Ceará, 620.010-560,
(E-mails: adrieloliveira@@hotmail.com,vandilberto@yahoo.com.br, camila_tabosa2@hotmail.com, ligiarmachado3@gmail.com, alexsandrofreitas2009@hotmail.com ).

\begin{abstract}
The quadrotors have been widely used in a number of applications, and studies on them are expanded. The monitoring of the energy availability of four-cylinder batteries is carried out based on knowledge related to the discharge curve of these energy storage devices. This work presents the application of structures of linear parametric models of the theory of systems identification, , with the objective of identifying the mathematical model that best represents the dynamics of discharge of batteries used in Rolling Spider quadrotors. The ARX, ARMAX, and BJ model structures were applied from data collected in real experiments. The models were implemented with the aid of the computational tool ident, present in Matlab/Simulink software. The results obtained prove that the parametric models used are effective to describe the discharge profile of the batteries used, and the BJ model presented a better result when compared to the other models.

Resumo: Os quadrirotores tem sido largamente utilizados em diversas aplicações, fazendo com que os estudos acerca destes sejam expandidos. O monitoramento da disponibilidade energética das baterias dos quadrirotores é realizado com base em conhecimentos relacionados a curva de descarga desses dispositivos armazenadores de energia. Este trabalho apresenta a aplicação de estruturas de modelos paramétricos lineares da teoria de identificação de sistemas, com o objetivo de identificar o modelo matemático que melhor representa a dinâmica de descarga de baterias utilizadas em quadrirotores Rolling Spider. Foram aplicadas as estruturas de modelos ARX, ARMAX, e BJ a partir de dados coletados em experimentos reais. Os modelos foram implementados com o auxílio da ferramenta computacional ident, presente no software Matlab/Simulink. Os resultados obtidos comprovam que os modelos paramétricos utilizados são eficazes para descrever o perfil de descarga das baterias utilizadas, sendo que o modelo BJ apresentou um melhor resultado quando comparado com os demais modelos.
\end{abstract}

Keywords: Quadrotors, Systems Identification, Mathematical Modeling, Discharge Curve. Palavras-chaves: Quadrirotores, Identificação de Sistemas, Modelagem Matemática, Curva de Descarga.

\section{INTRODUÇÃO}

No cenário da aviação, diversos veículos aéreos têm sido desenvolvidos para uso civil e militar, contudo, a presença de um piloto em tais veículos sempre era obrigatória. Com o avanço tecnológico, sistemas de alta complexidade foram desenvolvidos, possibilitando o controle das aeronaves de forma remota ou autônoma. Surge, então, os veículos aéreos não tripulados(VANTs), uma aeronave munida de um sistema embarcado inteligente ligado a diversos sensores e atuadores, tornando esse veículo capaz de realizar diversas atividades com objetivo pessoal ou profissional(Silva and Cordeiro, 2011).
Além do desenvolvimento de protótipos de baixo custo, outros dois fatores contribuíram de forma significativa para o aumento da utilização de quadrirotores em diversas áreas. $\mathrm{O}$ primeiro fator consiste no aumento da contribuição de pesquisadores para o controle de trajetória e estabilidade deste tipo de aeronave(Silva, 2015). Já o segundo fator consiste na versatilidade obtida a partir do auxílio de uma fonte de energia denominada bateria(Romio et al., 2012). No projeto de quadrirotores o tempo de vida da bateria é considerado uma das características mais importantes, pois indica o tempo que a aeronave irá operar sem a necessidade de conectar a uma fonte externa. 
A autonomia de voo deste tipo de aeronave está intrinsecamente ligado às limitações de tamanho, peso e capacidade de armazenamento da bateria acoplada em sua fuselagem. Assim é notório a necessidade, por parte dos fabricantes de baterias desenvolverem produtos que atendam a essas limitações e proporcionem uma maior capacidade de armazenamento(Romio, 2013), consequentemente uma maior segurança e autonomia de voo.

Outra necessidade importante é a utilização de métodos capazes de realizar o prognóstico do tempo de vida de baterias, para a referida predição utilizam-se diversas técnicas para modelar as características de descarga de uma bateria, os modelos matemáticos com amplo destaque na literatura são os de enfoque analítico, elétrico, estocástico, híbrido e experimental(Douglas et al., 2018). O método experimental não tem sido utilizado no contexto de VANTs, por ser inviável do ponto de vista econômico, e pelo motivo apresentar dificuldades na implementação(Romio, 2013), pois o tempo de vida da bateria dos quadrirotores dependem também de fatores externos que atuam no sistema dinâmico da aeronave através de distúrbios.

Neste contexto, o presente trabalho tem como objetivo aplicar técnicas de identificação de sistemas para obter um modelo acurado e versável que caracterize a dinâmica de descarga da bateria de um VANT do tipo quadrirotor. Para tanto, será apresentado o estudo e aplicação dos modelos paramétricos lineares ARX,ARMAX e BJ a partir de dados obtidos em experimentos reais.

Assim, o artigo está organizado da seguinte forma. Na Seção 2 são descritos alguns conceitos importantes relacionados à baterias. Na Seção 3 são apresentados os modelos paramétricos lineares da teoria de identificação de sistemas que foram utilizados no presente trabalho, já na Seção 4 são apresentados a plataforma de testes utilizada para aquisição dos dados experimentais, além dos procedimentos utilizados para coletar os dados e estimação dos parâmetros. Na Seção 5 são apresentados os parâmetros estimados, a validação dos modelos matemáticos ARX,ARMAX e BJ, e a acurácia apresentada por cada modelo. E finalmente, na Seção 6 é apresentada a conclusão.

\section{BATERIAS}

Uma bateria é constituída de células eletroquímicas que podem ser conectadas em série, paralelo, e em ligações mistas. Nessas células ocorre o processo de reação eletroquímica e com isso a energia química armazenada nas células é transformada em energia elétrica. O esquema de composição de uma célula eletroquímica(Figura 1) é formada por dois eletrodos: ânodo e cátodos, e entre esse eletrodos tem um eletrólito que os separa(Romio, 2013).

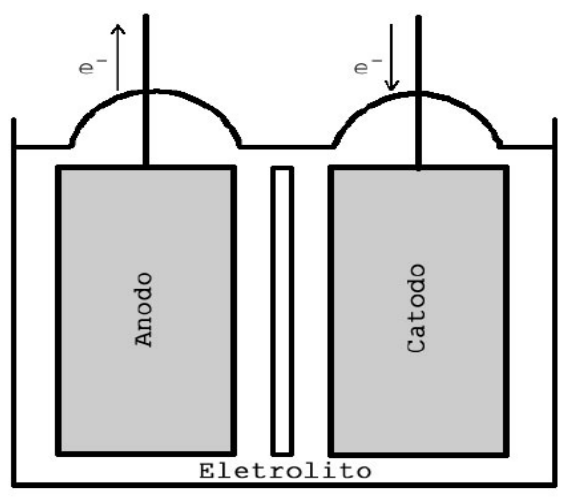

Figura 1. Esquema de composição de uma célula eletroquímica.

Quando ocorre o processo de descarga da bateria, na parte do ânodo acontece uma reação de oxidação, e na do cátodo o processo é uma reação de redução. Devido esses processos é que ocorre a troca de elétrons na célula eletroquímica. No processo de oxiredução, a Tensão (expressa em volts) e a capacidade (expressa em ampere-hora) são grandezas, que quando estudadas e feito o produto delas é possível mensurar o quanto de energia armazenada tem disponível na bateria. Considerando a grandeza Tensão no caso ótimo de descarga de uma bateria ideal é constante durante o período de descarregamento e tem uma queda instantânea a zero quando a bateria fica descarregada, com isso a capacidade ideal é constante no processo de descarga e toda a energia armazenada é utilizada no processo. Mas quando se trata de um caso real, aparecem variáveis nãolineares que devem ser estudadas pois elas influenciam no tempo de vida útil da bateria.

\subsection{Efeito de Recuperação}

Um dos efeitos não-lineares que devem ser estudados é o Efeito de Recuperação, que ocorre em períodos de relaxação da bateria, onde há pouca energia para ser escoada. Nesse período ocorre uma reestruturação dos elétrons nas células e com isso um aumento da capacidade da bateria, isso acontece quando se tem uma quantidade significativa de carga antes do sistema chegar no nível do cutoff. Na Figura 2, pode ser observados os estágios do efeito de recuperação da bateria, onde em 'A' a bateria está na sua totalidade de carga, onde pode ser observado que em todo comprimento do eletrólito se tem um agrupamento de espécies eletroativas. Em 'B' acontece um descarregamento, onde é diminuída as espécies eletroativas junto ao eletrodo. Depois disso, ocorre o momento de relaxação da bateria, que acontece quando a corrente de descarga se atenua, onde acontece a reestruturação dos elétrons, em 'C'. Assim, aumenta o número de espécies eletroativas próximas ao eletrodo novamente, até o gradiente de concentração ficar inexistente, com isso a capacidade efetiva da bateria é ampliado, onde pode ser visto em ' $\mathrm{D}$ '. Contudo, a quantidade de espécie eletroativas não consegue ser da mesma quantidade inicial, sendo menor do que tinha no início do processo. Em 'E' quando a bateria atinge um estágio inferior a carga, as reações eletroquímicas se findam e a bateria chega ao estágio de descarga(Romio et al., 2012). 


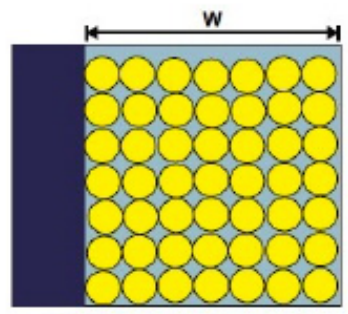

(A) Totalmente Carregada

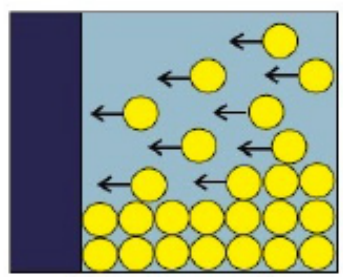

(C) Em Recuperação

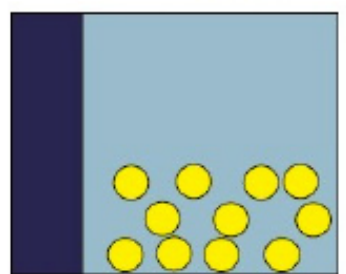

(E) Bateria Descarregada

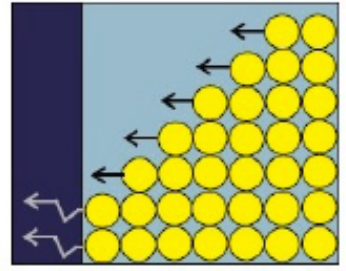

(B) Descarregando

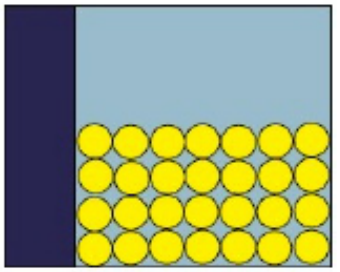

(D) Após Recuperação

\section{Legenda}

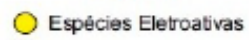

$\leftarrow$ Difusão

th. Consumo

$\square$ Eletrólito

$\square$ Eletrodo
Figura 2. Estágios de operação da bateria.

\subsection{Nivel de Cutoff}

Outro efeito não-linear que deve ser estudados é o Nível de Cutoff, ele é útil para estimar o tempo de vida da bateria. O nível de cutoff pode ser definido como o nível de capacidade mínima da bateria, pois toda bateria tem um nível de tensão para se manter funcionando e quando esse nível de tensão é atingido a bateria perde a funcionalidade e não consegue mais realizar as reações eletroquímicas nas células. Esse nível de cutoff não leva em consideração a descarga total, mas sim a possibilidade de manter as reações nas células.

\subsection{Efeito de Taxa de Capacidade}

O Efeito de Taxa de Capacidade também deve ser analisado, esse efeito leva em consideração a capacidade atual da bateria e a intensidade de corrente de descarregamento. Quando se tem correntes elevadas de descargas a capacidade efetiva é baixa, pois não tem tempo hábil para a reorganização das células, que é o Efeito de Recuperação. Já quando se tem cargas alternadas a capacidade efetiva aumenta, pois quando se tem um período sem fluxo de corrente os elétrons fazem um processo de reorganização aumentando assim a quantidade de carga na superfície do eletrodo. E assim aumentando a capacidade da bateria e por consequência um aumento da quantidade de energia disponível na bateria.

\section{IDENTIFICAÇÃO DE SISTEMAS}

Na literatura técnica há dois tipos de abordagem comumente utilizadas para modelar matemáticamente a dinâmica de um sistemas, sendo elas: a modelagem conceitual, e por identificação de sistemas(Romio et al., 2012). A abordagem conceitual é também conhecida como modelagem pela física ou natureza do processo, nela é necessário conhecer bem o sistema de estudo, bem como as leis da física que regem o sistema a ser modelado(Aguirre, 2007).

Conforme Aguirre (2007), na abordagem por identificação de sistemas é possível realizar a modelagem matemática tendo pouco ou nenhum conhecimento prévio do sistema. Nesta abordagem, quando não há nenhuma informação da física do sistema a identificação é denominada caixa preta. Já quando há alguma informação auxiliar, diferente das disponíveis no banco de dados utilizados, a identitificação é denominada caixa cinza. Contudo pode-se analisar um sistema dinâmico no domínio do tempo ou no domínio da frequência, logo a identificação pode derivar modelos (lineares ou não lineares).

No presente trabalho, para realizar a previção da curva de descarga da bateria de um quadrirotor Rolling Spider, serão utilizados os modelos matemáticos paramétricos lineares ARX (Auto regressivo com entradas exógenas), ARMAX (Auto regressivo com média móvel e entradas exógenas), e BJ (Box Jenkins).

A estrutura geral representada em tempo discreto é dada pela seguinte equação(Aguirre, 2007, p. 125):

$$
A(q) y(k)=\frac{B(q)}{F(q)} u(k)+\frac{C(q)}{D(q)} v(k)
$$

Onde: $q^{-1}$ é o operador de atraso, de modo que $y(k) q^{-1}=$ $y(k-1)$, e $v(k)$ é considerado o ruído branco e $A(q), B(q)$, $C(q), D(q)$ e $F(q)$ representam os polinômios definidos a seguir:

$$
\begin{gathered}
A(q)=1-a_{1} q^{-1}-\ldots-a_{n_{y}} q^{-n_{y}} \\
B(q)=b_{1} q^{-1}+\ldots+b_{n_{u}} q^{-n_{u}} \\
C(q)=1+c_{1} q^{-1}+\ldots+c_{n_{v}} q^{-n_{v}} \\
D(q)=1+d_{1} q^{-1}+\ldots+d_{n_{d}} q^{-n_{d}} \\
F(q)=1+f_{1} q^{-1}+\ldots+f_{n_{f}} q^{-n_{f}}
\end{gathered}
$$

Com base em (1), realizando devidas modificações, é possível obter os principais modelos paramétricos lineares, conforme descrito a seguir(Romio et al., 2012):

\subsection{Modelo ARX}

O modelo ARX (do inglês: Autoregressive with exogenous inputs), pode ser obtido a partir de (1), adotando $C(q)=$ $D(q)=F(q)=1$ sendo $A(q)$ e $B(q)$ polinômios arbitrários, tendo como resultado:

$$
A(q) y(k)=B(q) u(k)+v(k)
$$

A Figura 3 mostra a representação esquemática do modelo ARX, utilizando diagrama de blocos.

\subsection{Modelo ARMAX}

O modelo ARMAX (do inglês Autoregressive moving verage with exogenous input), pode ser obtido a partir de (1), 


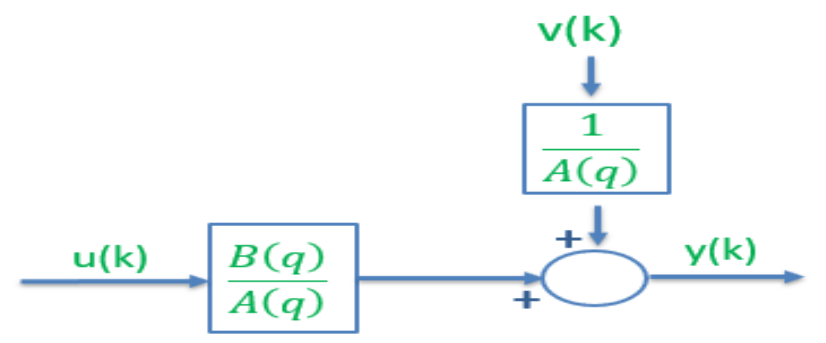

Figura 3. Representação esquemática do modelo ARX.

adotando $D=(q)=F(q)=1$ e $A(q), B(q)$ e $C(q)$ como polinômios arbitrários, tendo como resultado:

$$
A(q) y(k)=B(q) u(k)+C(q) v(k)
$$

A Figura 4 mostra a representação esquemática do modelo ARMAX, utilizando diagrama de blocos.

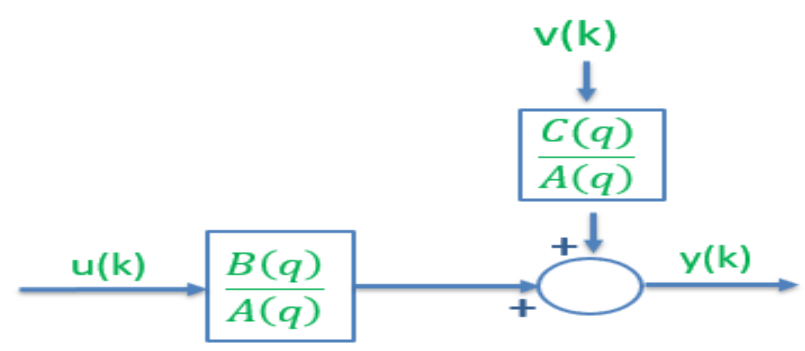

Figura 4. Representação esquemática do modelo ARMAX.

\subsection{Modelo BJ}

O modelo BJ(do inglês: Box-Jenkins) é obtido a partir de (1), adotando $A(q)=1$. Todos os demais polinômios são arbitrários, obtendo como resultado:

$$
y(k)=\frac{B(q)}{F(q)} u(k)+\frac{C(q)}{D(q)} v(k)
$$

A Figura 5 mostra a representação esquemática do modelo BJ, utilizando diagrama de blocos.

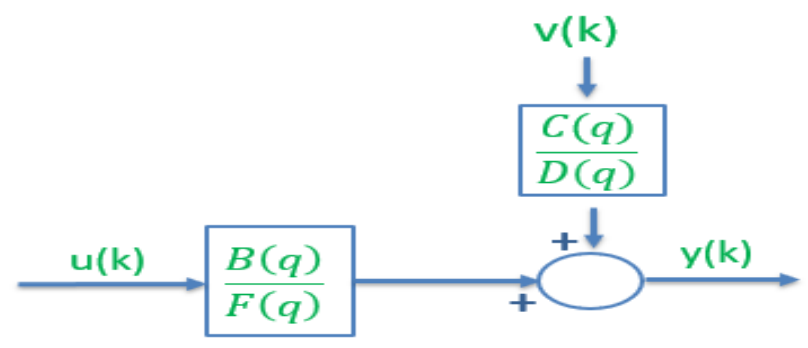

Figura 5. Representação esquemática do modelo BJ.

\section{MATERIAIS E MÉTODOS}

Nesta seção é apresentada a plataforma utilizada para realizar os ensaios e aquisição de dados característicos da descarga da bateria de um drone do tipo quadrirotor, assim como os materiais utilizados. Em seguida são apresentados os bancos de dados utilizados para estimar os parâmetros, e validar os modelos matemáticos : ARX, ARMAX, e BJ obtidos a partir da teoria de identificação de sistemas.

\subsection{Plataforma de testes}

Os dados apresentados neste trabalho foram obtidos a partir de uma plataforma de testes composta por um drone Parrot Rolling Spider, um Dongle Bluetooth USB 4.0, e um notebook em conjunto com o software Matlab/Simulink. O Dongle USB é responsável por estabelecer uma conexão bluetooth entre o notebook e o hardware do drone. Já o software Matlab/Simulink é utilizado para criar, modificar e enviar algoritmos de controle de voo e aquisição de dados do perfil de descarga da bateria, através da conexão da conexão bluetooth estabelecida. A Figura 6 a seguir apresenta uma foto do ambiente de testes:

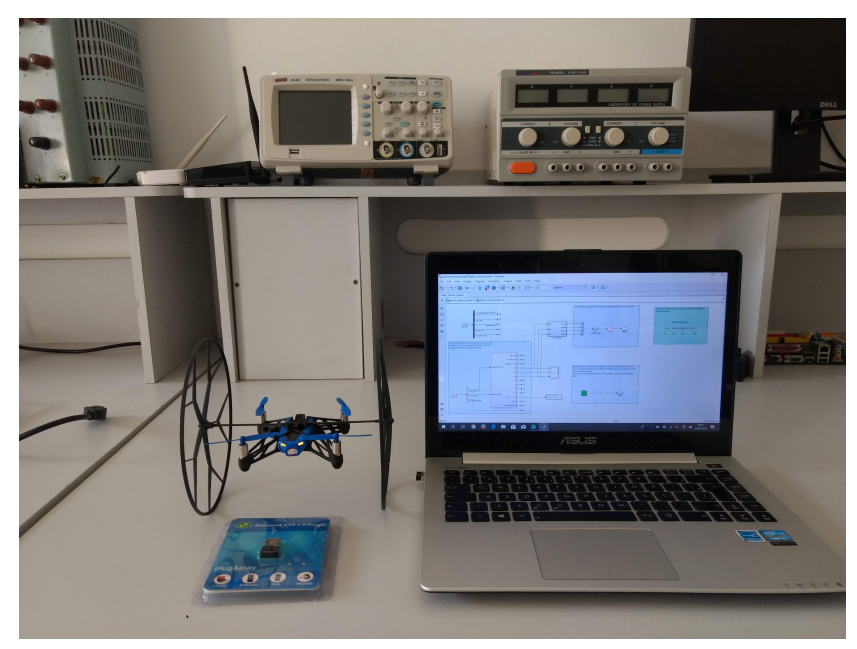

Figura 6. Plataforma de Testes.

\subsection{Ensaios e aquisição dos dados experimentais}

Os ensaios foram realizados mantendo o drone pairando, onde simula a descarga da bateria em corrente constante. Para manter o quadrirotor pairando, e ao mesmo tempo utilizá-lo como uma carga não variável, é necessário que a velocidade de giro dos quatro propulsores sejam iguais durante todo processo de descarga.

Para a aquisição de dados foi utilizado o seguinte procedimento. Inicialmente as baterias foram submetidas a um processo de carga completo, conectando-as em uma fonte de carregamento externa . Após este procedimento, as baterias são desconectadas da fonte de carga externa e conectadas ao drone. Em seguida foi estabelecido uma conexão bluetooth entre o notebook e o quadrirotor. Por fim, algoritmos de controle e aquisição de dados foram enviados para o hardware do veículo aéreo com o objetivo de estabelecer uma velocidade de giro constante nos quatro propulsores, e registrar em tempo real os valores de tensão nos terminais da bateria. A Figura 7 mostra o diagrama de blocos utilizado para controlar a velocidade dos propulsores, e obter os dados do comportamento de descarga da bateria.

Os ensaios são realizados considerando 2 baterias de Li-Po novas da marca Shoot, modelo XT-412. Para cada bateria 


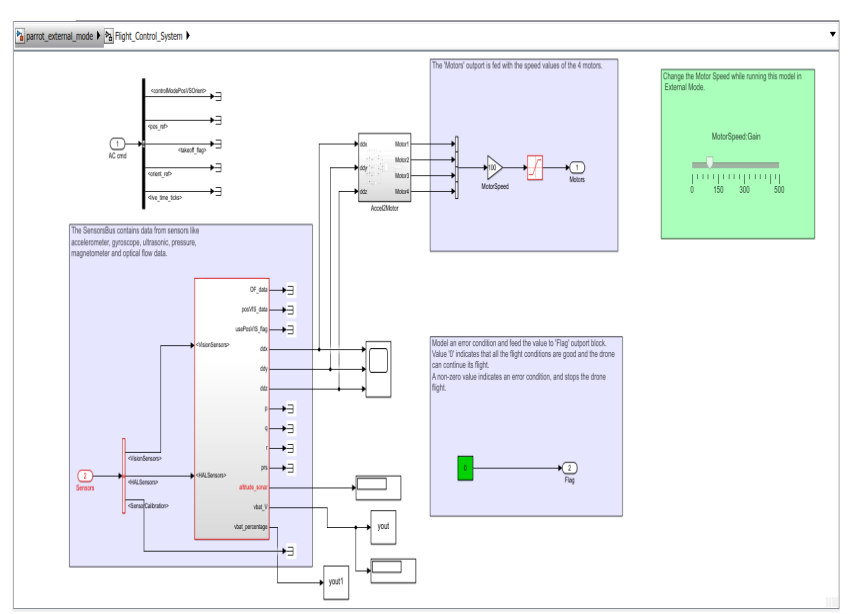

Figura 7. Diagrama de blocos utilizado.

são realizados 5 ensaios, em cada um deles é registrado o perfil de descarga da bateria variando de $3,30 \mathrm{~V}$ a $4,10 \mathrm{~V}$, em intervalos de $50(\mathrm{mV})$. Vale ressaltar que, para o veículo aéreo utilizado, o nível $0 \%$ de carga da bateria é mensurado em 3,20V. Já o nível 100\% de carga da bateria é mensurado em $4,2 \mathrm{~V}$.

Os dados experimentais obtidos neste processo são divididos em dois conjuntos de ensaios. O primeiro conjunto é formado pelos resultados dos 5 ensaios experimentais realizados com a Bateria A, é utilizado para estimar os parâmetros dos modelos ARX, ARMAX, e BJ. Já o segundo conjunto é formado pelos resultados dos 5 ensaios experimentais realizados com a Bateria B, sendo utilizado para a validação dos modelos supracitados.

As Tabelas 1 e 2 apresentam os dados experimentais do primeiro e segundo conjunto de ensaios respectivamente, onde $T B_{e i}$ é o tempo de vida experimental, $i$ caracteriza o número do experimento, e $T B_{e m}$ é o tempo de vida experimental médio, sendo apresentados em minutos.

Tabela 1. Dados experimentais do primeiro conjunto de ensaios.

\begin{tabular}{c|c|c|c|c|c|c}
\hline $\begin{array}{c}\text { Tensão } \\
(\mathrm{V})\end{array}$ & $\begin{array}{c}T B_{e 1} \\
(\mathrm{~min})\end{array}$ & $\begin{array}{c}T B_{e 2} \\
(\mathrm{~min})\end{array}$ & $\begin{array}{c}T B_{e 3} \\
(\mathrm{~min})\end{array}$ & $\begin{array}{c}T B_{e 4} \\
(\mathrm{~min})\end{array}$ & $\begin{array}{c}T B_{e 5} \\
(\mathrm{~min})\end{array}$ & $\begin{array}{c}T B_{e m} \\
(\mathrm{~min})\end{array}$ \\
\hline 3.30 & 13,95 & 13,43 & 13,30 & 11,74 & 11,83 & 12,85 \\
\hline 3.35 & 13,88 & 13,38 & 13,24 & 11,68 & 11,77 & 12,79 \\
\hline 3.40 & 13,79 & 13,28 & 13,16 & 11,60 & 11,68 & 12,70 \\
\hline 3.45 & 13,66 & 13,17 & 13,07 & 11,50 & 11,58 & 12,60 \\
\hline 3.50 & 13,55 & 13,03 & 12,92 & 11,37 & 11,46 & 12,46 \\
\hline 3.55 & 13,37 & 12,86 & 12,75 & 11,21 & 11,30 & 12,30 \\
\hline 3.60 & 13,08 & 12,57 & 12,47 & 10,74 & 10,89 & 11,95 \\
\hline 3.65 & 11,82 & 11,26 & 10,89 & 9,49 & 9,62 & 10,62 \\
\hline 3.70 & 9,46 & 8,94 & 9,12 & 7,49 & 7,38 & 8,48 \\
\hline 3.75 & 7,58 & 6,94 & 7,28 & 5,76 & 5,79 & 6,67 \\
\hline 3.80 & 5,84 & 5,66 & 5,79 & 4,58 & 4,59 & 5,29 \\
\hline 3.85 & 4,57 & 4,47 & 4,38 & 3,56 & 3,51 & 4,10 \\
\hline 3.90 & 3,63 & 3,22 & 3,49 & 2,66 & 2,65 & 3,13 \\
\hline 3.95 & 2,70 & 2,57 & 2,67 & 2,06 & 2,15 & 2,43 \\
\hline 4.00 & 2,11 & 2,01 & 1,99 & 1,39 & 1,37 & 1,77 \\
\hline 4.05 & 1,23 & 0,99 & 1,10 & 0,77 & 0,73 & 0,96 \\
\hline 4.10 & 0,48 & 0,35 & 0,39 & 0,17 & 0,20 & 0,32 \\
\hline
\end{tabular}

Tabela 2. Dados experimentais do segundo conjunto de ensaios.

\begin{tabular}{c|c|c|c|c|c|c}
\hline $\begin{array}{c}\text { Tensão } \\
(\mathrm{V})\end{array}$ & $\begin{array}{c}T B_{e 1} \\
(\min )\end{array}$ & $\begin{array}{c}T B_{e 2} \\
(\min )\end{array}$ & $\begin{array}{c}T B_{e 3} \\
(\min )\end{array}$ & $\begin{array}{c}T B_{e 4} \\
(\min )\end{array}$ & $\begin{array}{c}T B_{e 5} \\
(\min )\end{array}$ & $\begin{array}{c}T B_{e m} \\
(\mathrm{~min})\end{array}$ \\
\hline 3,32 & 12,63 & 12,54 & 12,47 & 11,77 & 11,42 & 12,17 \\
\hline 3,37 & 12,53 & 12,43 & 12,39 & 11,67 & 11,34 & 12,07 \\
\hline 3,42 & 12,44 & 12,33 & 12,28 & 11,57 & 11,24 & 11,97 \\
\hline 3,47 & 12,27 & 12,18 & 12,12 & 11,43 & 11,11 & 11,82 \\
\hline 3,52 & 12,11 & 11,98 & 11,93 & 11,24 & 10,94 & 11,64 \\
\hline 3,57 & 11,84 & 11,74 & 11,63 & 10,96 & 10,60 & 11,35 \\
\hline 3,62 & 11,33 & 11,03 & 11,03 & 10,01 & 9,89 & 10,66 \\
\hline 3,67 & 9,81 & 9,56 & 9,42 & 8,70 & 8,54 & 9,21 \\
\hline 3,72 & 7,91 & 7,67 & 7,58 & 7,29 & 6,56 & 7,40 \\
\hline 3,77 & 6,26 & 6,18 & 6,01 & 5,75 & 5,33 & 5,91 \\
\hline 3,82 & 4,94 & 4,86 & 4,76 & 4,62 & 4,15 & 4,67 \\
\hline 3,87 & 3,88 & 3,91 & 3,83 & 3,74 & 3,10 & 3,69 \\
\hline 3,92 & 2,99 & 3,00 & 2,84 & 2,97 & 2,31 & 2,82 \\
\hline 3,97 & 2,48 & 2,41 & 2,26 & 2,13 & 1,66 & 2,19 \\
\hline 4,02 & 2,15 & 1,59 & 1,42 & 1,33 & 0,85 & 1,47 \\
\hline 4,07 & 0,93 & 0,92 & 1,02 & 0,66 & 0,36 & 0,78 \\
\hline
\end{tabular}

\section{RESULTADOS E DISCUSSÕES}

A partir dos dados experimentais apresentados na Tabela 1 é realizado a modelagem matemática da curva que representa a dinâmica de descarga das baterias, utilizando a teoria de identificação de sistemas. Os modelos paramétricos lineares foram obtidos utilizando o toolbox ident do software Matlab/Simulink. A seguir são apresentados os polinômios e equações correspondentes aos modelos paramétricos lineares ARX, ARMAX e BJ, obtidos a partir do toolbox ident:

Modelo ARX: Para este modelo foram estimados as seguintes polinômios:

$$
\begin{gathered}
A(q)=1-1.523 q^{-1}+0.7659 q^{-2} \\
B(q)=376.8 q^{-1}-381.5 q^{-2}
\end{gathered}
$$

onde $q^{-1}$ é o operador de atraso, conforme Aguirre (2007). Pode-se escrever a equação do modelo ARX (7) da seguinte forma:

$$
\begin{aligned}
& y(k)=\frac{376.8 q^{-1}-381.5 q^{-2}}{1-1.523 q^{-1}+0.7659 q^{-2}} u(k)+ \\
& \frac{1}{=1-1.523 q^{-1}+0.7659 q^{-2}} v(k)
\end{aligned}
$$

Modelo ARMAX: Para este modelo foram estimados as seguintes polinômios:

$$
\begin{gathered}
A(q)=1-1.741 q^{-1}+0.9195 q^{-2} \\
B(q)=263.7 q^{-1}-267 q^{-2} \\
C(q)=1-0.04337 q^{-1}-0.9566 q^{-2}
\end{gathered}
$$

Pode-se escrever a equação do modelo ARMAX (8) da seguinte forma:

$$
\begin{gathered}
y(k)=\frac{263.7 q^{-1}-267 q^{-2}}{1-1.741 q^{-1}+0.9195 q^{-2}} u(k)+ \\
\frac{1-0.04337 q^{-1}-0.9566 q^{-2}}{1-1.741 q^{-1}+0.9195 q^{-2}} v(k)
\end{gathered}
$$


Modelo BJ: Para este modelo foram estimados as seguintes polinômios:

$$
\begin{gathered}
B(q)=710 q^{-1}-718.8 q^{-2} \\
C(q)=1+0.8084 q^{-1}-0.1916 q^{-2} \\
D(q)=1-0.3873 q^{-1}+0.2052 q^{-2} \\
F(q)=1-1.389 q^{-1}+0.805 q^{-2}
\end{gathered}
$$

Pode-se escrever a equação do modelo BJ (9) da seguinte forma:

$$
\begin{gathered}
y(k)=\frac{710 q^{-1}-718.8 q^{-2}}{1-1.389 q^{-1}+0.805 q^{-2}} u(k)+ \\
\frac{1+0.8084 q^{-1}-0.1916 q^{-2}}{1-0.3873 q^{-1}+0.2052 q^{-2}} v(k)
\end{gathered}
$$

Após a determinação dos modelos matemáticos que caracterizam a dinâmica de descarga da bateria de Vant do tipo quadrirotor, conforme (10), (11), e (12) é necessário realizar a validação de cada modelo. A própria ferramenta ident utilizada neste trabalho, dispõe de um ambiente para realizar a validação, para isto serão utilizados os dados experimentais apresentados na Tabela 2. Na Figura 8 são apresentadas as curvas dos resultados da validação dos três modelos matemáticos ARX, ARMAX, e BJ.

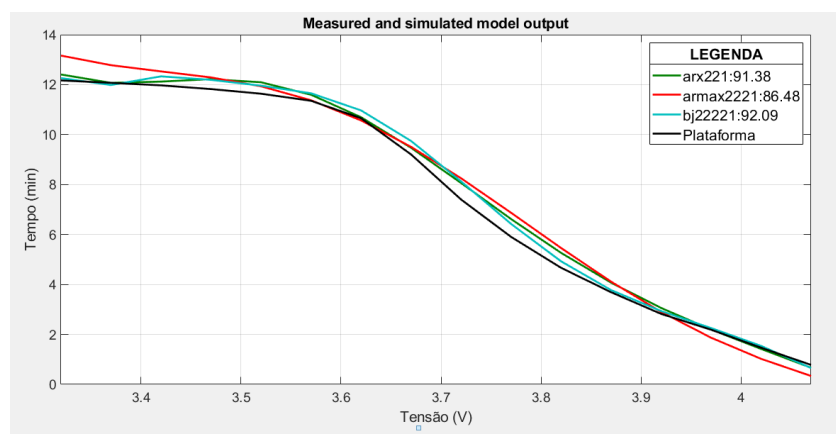

Figura 8. Resultados da validação dos modelos.

A partir da análise comparativa dos resultados apresentados na Figura 8, observa-se que a estrutura de modelo paramétrico BJ apresentou um melhor resultado quando comparado com os demais modelos simulados, ou seja, com o modelo BJ foi obtida uma acurácia de 92,09\%, já comos modelos ARX e ARMAX a acurácia obtida foi de $91.38 \%$ e $86.48 \%$ respectivamente.

\section{CONCLUSÃO}

Neste trabalho foi realizado a modelagem matemática caixa cinza, da curva de descarga de baterias que alimentam um quadrirotor Rolling Spider, utilizando conceitos da teoria de identificação de sistemas e uma plataforma de testes e aquisição de dados composta por um drone Rolling Spider, um adaptador bluetooth 4.0, e um leptop em conjunto com o software Matlab/Simulink. As baterias utilizadas no processo de descarga, na plataforma de testes, são de Li-Po, da marca Shoot, modelo XT-412. A partir da ferramenta ident, foram obtidos os parâmetros dos modelos lineares ARX, ARMAX, e BJ que caracterizam a dinâmica de descarga das baterias utilizadas. A partir da análise dos resultados observou-se que o modelo paramétrico linear Box Jenkins apresentou um melhor resultado quando comparado com os demais modelos e os dados experimentais, com uma acurácia de $92.09 \%$.

\section{AGRADECIMENTOS}

Este trabalho foi realizado durante um projeto de pesquisa na Universidade Federal do Ceará. Este trabalho recebeu apoio da Fundação Cearense de Apoio ao Desenvolvimento Científico e Tecnológico(FUNCAP).

\section{REFERÊNCIAS}

Aguirre, L. (2007). Introdução à identificação de sistemas. UFMG, Belo Horizonte.

Douglas, J.B.F., Julia, G.Z., Airam, T.Z.R.S., and Paulo, S.S. (2018). Identificação de sistemas aplicada à predição do tempo de vida de baterias de li-po utilizadas em dispositivos móveis. Proceeding Series of the Brazilian Society of Computational and Applied Mathematics, São José dos Campos. doi:10.5540/03.2018.006.01.0377.

Porciuncula, C.M.D. (2012). Aplicação de modelos elétricos de bateria na predição do tempo de vida de dispositivos móvei 2012. $74 \mathrm{f}$. Mestrado em modelagem matemática, Programa de Pós-Graduação em Modelagem Matemática, Universidade Regional do Noroeste do Estado do Rio Grande do Sul, Rio Grande do Sul.

Romio, L.C. (2013). Modelagem matemática do tempo de vida de baterias utilizando a teoria de identificação de sistemas 2013. $84 \mathrm{f}$. Mestrado em modelagem matemática, Programa de Pós-Graduação em Modelagem Matemática, Universidade Regional do Noroeste do Estado do Rio Grande do Sul, Rio Grande do Sul.

Romio, L.C., Sausen, A.T.Z.R., Sausen, P.S., and Reimbold, M.M.P. (2012). Aplicação de identificação de sistemas no tempo de vida de baterias de dispositivos móveis. 18-33. Revista de Ciências Exatas e Engenharias, Rio Grande do Sul.

Silva, L.P. and Cordeiro, C.S. (2011). Quadricóptero, aspectos gerais e análises da propulsão elétrica. In VIII Simpósio de Excelência em Gestão e Tecnologia, 14. Instiruto Federal Fluminense, Espirito Santo.

Silva, L.T.G. (2015). Modelagem, simulação e controle de um vant quadrirrotor. 